\title{
Super-Quantum Correlations: A Necessary Clarification
}

\author{
Pierre Uzan \\ Laboratory Sphere, Paris Diderot University, Paris, France \\ Email: pierre.uzan@paris7.jussieu.fr
}

How to cite this paper: Uzan, P. (2018) Super-Quantum Correlations: A Necessary Clarification. Journal of Quantum Information Science, 8, 131-137.

https://doi.org/10.4236/jqis.2018.83009

Received: August 13, 2018

Accepted: September 8, 2018

Published: September 11, 2018

Copyright (C) 2018 by author and Scientific Research Publishing Inc. This work is licensed under the Creative Commons Attribution International License (CC BY 4.0).

http://creativecommons.org/licenses/by/4.0/

\begin{abstract}
This paper proposes a necessary clarification about the problematic of super-quantum correlations, whose mainstream debate relies on an incorrect, statistical interpretation of the no-signaling condition. The no-signaling condition is an informational constraint that limits the strength of non-local correlations to the Tsirelson bound.
\end{abstract}

\section{Keywords}

Super-Quantum Correlations, No-Signaling, Information Causality

\section{Introduction}

It has been suggested that non-local correlations stronger than quantum correlations between two sub-systems that cannot exchange any information would be "theoretically possible" [1] [2]. The present paper will closely examine this assertion, the assumptions on which it is based and the mainstream argumentation of this question, where bipartite correlations are modelled in terms of "boxes". A "box", which is the central device of the Bell's game played by two parties, can be described by an arithmetic relation between couples of "inputs", which can be regarded as the indexes of the two directions (right or left) each of the two parties (Alice and Bob) push her/his joystick, and "outputs", which are the possible responses of the box for these actions. This convenient representation of bipartite correlations will be adopted here for discussing the validity of some mainstream ideas about the question of super-quantum correlations.

\section{Why No-Signaling Super-Quantum Correlations Would Be "Theoretically Possible"?}

1) The assumption according to which these correlations are more non-local 
than quantum correlations (for "super-quantum") is expressed, in Popescu's and Rohrlich's previously mentioned work, by the condition that the CHSH correlation factor between Alice's outcomes and Bob's outcomes violates the Tsirelson bound. The considered situation features the famous scenario proposed by Clauser, Horne, Shimony and Holt [3] in order to confirm experimentally Bell's theorem ${ }^{1}$. Alice and Bob, which cannot communicate (which means that no message can be exchanged according to relativistic causality), have to choose between two possible actions, indexed by $x=0$ or $x=1$ for Alice and by $y=0$ or $y=1$ for Bob. Each of these actions have binary outcomes: Alice's possible outcomes $\mathrm{A}_{\mathrm{x}}$ for the action $\mathrm{x}$ are $a=0$ or $a=1$, and Bob's possible outcomes $\mathrm{B}_{\mathrm{y}}$ for the action y are $b=0$ or $b=1$. The CHSH correlation factor between Alice's and Bob's outcomes is defined as:

$$
R=C\left(A_{0}, B_{0}\right)+C\left(A_{0}, B_{1}\right)+C\left(A_{1}, B_{0}\right)-C\left(A_{1}, B_{1}\right)
$$

where the $C\left(A_{x}, B_{y}\right) \equiv C_{x y}$ denote, for all $\mathrm{x}$ and $\mathrm{y}$, the correlation degree between the outcomes $A_{x}=a$ and $B_{y}=b$ for the joint action $(x, y)$. The magnitude of the $\mathrm{CHSH}$ correlation factor can inform us about the nature of the considered correlations: if $R \leq 2$, which is the Bell bound, these correlations are classical correlations that can be explained by their local properties; the case $2<R \leq 2 \sqrt{2}$, where $2 \sqrt{2}$ is the Tsirelson bound, describes quantum non-local correlations. The Tsirelson bound thus characterizes the maximal strength of quantum correlations [5], while its violation describes correlations that are stronger than the strongest quantum correlations:

$$
\text { (SQ) }|R|>2 \sqrt{2} \text {, }
$$

which defines "super-quantum" correlations according to the previously mentioned work of Popescu and Rohrlich [1] [2].

2) The "no-signaling" assumption (NS), which obviously aims to assert that Alice and Bob cannot exchange any signal or any information, has been expressed in the mainstream literature-for example in Popescu's and Rohrlich's work [2] - by the following statistical condition according to which the probability that Alice obtains a particular outcome " $a$ " is independent of the choice of Bob's action, when he decides to push his joystick to the right or to the left -that is, this probability is independent of the value of $y$-, and vice versa [1]:

For all possible actions $x, x^{\prime}, y, y^{\prime}$ and for all possible outcomes $a, b$,

$$
\sum_{b} P(a, b / x, y)=\sum_{b} P\left(a, b / x, y^{\prime}\right) \text { and } \sum_{a} P(a, b / x, y)=\sum_{a} P\left(a, b / x^{\prime}, y\right)
$$

Denoting the sum on the possible outcomes " $b$ " by $P(a / x, y)$ and the sum on the possible outcomes "a" by $P(b / x, y)$, this double condition, which interprets the (NS) assumption in terms of probabilities of outcomes, can be more conveniently reformulated as:

$$
\text { (NS) })_{\text {stat }} P(a / x, y)=P\left(a / x, y^{\prime}\right) \text { and } P(b / x, y)=P\left(b / x^{\prime}, y\right) \text {. }
$$

Section 3 will question this statistical interpretation of the no-signaling condi${ }^{1}$ Bell's theorem states that no local hidden variable theory can reproduce all the predictions of quantum mechanics [4]. 
tion by providing counter-examples that show that (NS) and (NS) stat $_{\text {are not sa- }}$ tisfied or falsified in the same time for some experimental situation.

3) Popescu and Rohrlich have shown that these two conditions (SQ) and (NS) $)_{\text {stat }}$ can be conjointly satisfied for some particular boxes, called PR-boxes, defined by the following relations [1]:

(PR) $a \oplus b=x \cdot y$, where “ $\oplus$ " is the addition modulo 2 , which can be rewritten in terms of probabilities of outcomes as follows [5]:

$$
P(a, b / x, y)=1 / 2 \text { if } a \oplus b=x \cdot y \text { is realized }=0 \text { otherwise. }
$$

Hence the generally undisputed assertion that no-signaling super-quantum correlations are "theoretically possible". However, such correlations whose existence would give rise to rather implausible consequences regarding the cost of distributed computation [6] have not been or not yet been observed. The discussion then concentrates on the possible reasons why the Tsirelson bound still holds, if that is the case-a discussion which is well illustrated by the evocative tittle of Bub's article "Why the Tsirelson bound?" [7].

\section{The Statistical Interpretation of the "No-Signaling" Condition Is Not Correct}

Due to the statistical interpretation (NS) stat of the no-signaling condition, it is then generally taken for granted that super-quantum correlations are "theoretically possible" and that only a meta-theoretical principle might ban this possibility. However, the latter reasoning is skewed by the incorrect probabilistic interpretation (NS) stat of the "no-signaling" assumption (NS), which obviously is an informational condition asserting that Alice and Bob do not exchange any information, neither through space nor by other means. As shown by the follow-

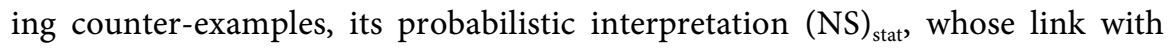
relativistic causality is already doubtful, is weaker than what (NS) requires.

\section{1. (NS) stat Does Not Correctly Express Relativistic Causality (RC)}

1) (RC) and no (NS) stat: Suppose that at each round, Alice sends Bob information about her input $\mathrm{x}$ at a speed smaller than the speed of light, and suppose that Bob systematically chooses the same input than Alice: $y=x$. In this situation, (NS) $)_{\text {stat }}$ is not satisfied since the probability that Bob gets the outcome $b$ given the input $\mathrm{y}$ depends on Alice's action: $p(b / y)=p(b / x)$. However, (RC) is satisfied since Alice has sent the information about her input at a speed smaller than the speed of light.

2) (NS) $)_{\text {stat }}$ and no (RC): Suppose that at each round it would be possible that Alice sends Bob information about her input $x$ at a speed greater than the speed of light, and suppose that Bob does not care about this information or cannot receive it (for example, because he can be blind or deaf) and that, consequently, his inputs and his outcomes can still be totally independent of Alice's inputs. In this situation, (RC) is obviously not satisfied since Alice's message has been sent at a speed greater than the speed of light but (NS) stat may still be satisfied. 


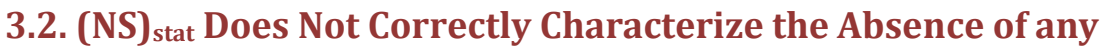 Exchange of Information}

1) (NS) $)_{\text {stat }}$ and no (NS): Consider a deterministic box, for which $x_{i}$ determines the outcome $a_{i}$ for Alice and $y_{j}$ determines the outcome $b_{j}$ for Bob and suppose that at each round Alice systematically informs Bob of her choice of action $\mathrm{x}$. In this situation, (NS) $)_{\text {stat }}$ is satisfied since $p\left(a_{i} / x_{i^{\prime}}, y\right)=p\left(a_{i} / x_{i^{\prime}}\right)=\delta_{i i^{\prime}}$, which is independent of $y$ and $p\left(b_{j} / x, y_{j^{\prime}}\right)=p\left(b_{j} / y_{j^{\prime}}\right)=\delta_{j j^{\prime}}$, which is independent of $x$. However, (NS) is obviously not satisfied since a signal has been sent from Alice to Bob.

2) The only valid deduction is that (NS) implies (NS) $)_{\text {stat }}$ if no information is exchanged between Alice and Bob, whatever the means, the probabilities of outcomes for a party do not a priori depend on the action performed by the other party: (NS) stat is then only a necessary condition of no-signaling (NS).

Thus, the statistical condition (NS) stat $i$ too weak to characterize the absence of any exchange of information (NS) and, moreover, it does not adequately express relativistic causality (RC). We then have to a priori reject this statistical interpretation of (NS)-unless we use (NS) $)_{\text {stat }}$ as a necessary (but not sufficient) condition of (NS) - along with the mainstream opinion according to which PR-boxes would be "theoretically" possible and could only be ruled out by a meta-theoretical principle.

\section{No-Signaling, Information Causality and the Tsirelson Bound}

To correctly interpret (NS), let's come back to the original meaning of the term "no-signaling": no signal or no information is exchanged, i.e., applied to the considered situation,

"Alice and Bob do not exchange any information, whatever means"

If Alice and Bob do not exchange any information, what Alice does (namely, choosing an input and reading the output) is independent of any experiment performed by Bob (and vice-versa). Note that by assuming that Alice's and Bob's inputs/outputs operations are experiments performed on two separated systems S1 and S2 respectively, this experimental independence echoes with Einstein's basic requirement of local realism for a composite system S1 + S2 that he expressed as follows [8]:

“...every statement regarding S2 which we are able to make on the basis of a complete measurement on S1 must hold for the system S2 if, after all, no measurement whatsoever ensued on S1."

Indeed, as upheld by Pawlowski, it seems obvious that the no-signaling condition (NS) is nothing but a particular specification (for $m=0$ ) of the Information Causality principle he has proposed [9]. The latter informational principle asserts that:

"Bob can gain no more than $\mathrm{m}$ (classical) bits of information about Alice's ${ }^{2}$ That the information sent by Alice to Bob is understood as being sent through space (and not by other means) is implicit all along Pawlowski's work [9]. For example, it is also clearly shown in the illustrative figure 1 of the quoted article. This essential point has been explicitly mentioned here. 
data if she sends him through space ${ }^{2}$ only $\mathrm{m}$ bits", which discards any non-local exchange of information; and the additive condition that $m=0$ discards any spatial exchange of information. Consequently, Information Causality with $\mathrm{m}$ equal to 0 , which can be noted as (IC), rules out any exchange of information, which is exactly the meaning of (NS).

Now, as shown by Pawlowski and colleagues [9], and also by Bub [7], the Tsirelson bound can be derived from Information Causality (IC). This derivation, which refers to a construction proposed by van Dam [6], evaluates the Shannon mutual information between Alice's data, which is a string of $\mathrm{N}$ random and independent bits $\left\{a_{1} \ldots, a_{k} \ldots, a_{N}\right\}$, and Bob's output, denoted as $\beta$, when Alice sends him a message of $m$ classical bits with the help of which Bob is required to guess the b-th bit of Alice's data:

$$
I \equiv \sum_{k=1 \text { to } N} I\left(a_{k}: b / b=k\right),
$$

and limits this mutual information to $\mathrm{m}: I \leq m$. The evaluation of this mutual information involves Bob's probabilities of success for guessing a specific bit in Alice's data, which are, in turn, involved in the CHSH correlation factor. This link between the latter mutual information and the $\mathrm{CHSH}$ correlation factor then allows to show that the Information Causality principle is violated as soon as the Tsirelson bound is [7] [9].

The existence of such a derivation of the Tsirelson bound from the only no-signaling condition (NS) $\equiv(\mathrm{IC})_{0}$, that is without using the quantum formalism of operators, obviously shows that the Tsirelson bound is a very general upper bound of the strength of all no-signaling correlations. Contrary to the mainstream opinion, the Tsirelson bound is NOT a specific "quantum" bound, it is a universal bound of the strength of correlations between two separated physical systems, where "separated" means that these systems cannot exchange any information or that, in other words, they obey to the no-signaling condition.

Thus, if we call "super-quantum correlations" correlations that are "stronger than the strongest quantum correlations" (see Section 2), it can be concluded that the no-signaling condition (NS), which is a particular specification (for $m=0$ ) of Information Causality, is sufficient to rule out the "theoretical possibility" of such super-quantum correlations. This conclusion contradicts the assertion by Popescu and Rorhlich (see Section 1) - an assertion based on a too weak, statistical interpretation of the no-signaling condition (NS).

Note that another interpretation of the term "super-quantum" has been provided in the literature [10] [11]: "super-quantum" or "post-quantum", would refer to the impossibility of describing an experimental situation within the framework of quantum theory, by representing observables by operators of a $\mathrm{C}^{*}$ algebra, states as vectors of a Hilbert space and by computing the probabilities of outcomes by the Born rule. With this linguistic-like interpretation of "super-quantum", as quantum describability, the latter conclusion does not hold any more because, as can be shown (see, for example, Ishizaka [12] or Allcock et al. [13]), quantum "noisy" states, which are partially entangled, can give rise to 
correlations whose maximal degree is smaller than the Tsirelson bound. This implies that the quantum describability interpretation of "super-quantum" does not coincide with Popescu's and Rohrlich's interpretation of "super-quantum" in terms of "stronger than quantum correlations", interpretation on which we have focused in this article. However, this interesting result does not question the proposed clarification of the notion of "no-signaling" which, if correctly interpreted as an informational condition, is then independent of the language that is used to describe a physical system. On the contrary, this result reinforces the idea supported in this article according to which the Tsirelson bound, which can be derived from the Information Causality principle, is not a specific quantum bound.

\section{Conclusion and Prospect}

We have shown that the no-signaling condition (NS) is not correctly interpreted by the usual statistical condition and should be regarded, as suggested by Pawlowski, as a specification of the Information Causality principle for which no information is exchanged through space. Consequently, when correctly interpreted, the no-signaling condition DOES NOT ALLOW the existence of correlations stronger than the strongest quantum correlations (that would violate the Tsirelson-bound) - and, in this sense of "super-quantum", super-quantum correlations cannot then exist, even "theoretically"!

Indeed, it seems that a genuine questioning of the limits of all the current theoretical representations of phenomena (including quantum theory) is a questioning of the Information Causality principle that governs these representations, which would allow non-local communication and would give rise to a rather amazing, but maybe real world that future research has to explore.

\section{Conflicts of Interest}

The authors declare no conflicts of interest regarding the publication of this paper.

\section{References}

[1] Popescu, S. (2015) Non-Locality beyond Quantum Mechanics. In: Bokulich, A. and Jaeger, G., Eds., Philosophy of Quantum Information and Entanglement, Cambridge Books Online, 3-15.

[2] Popescu, S. and Rohrlich, D. (1994) Quantum Nonlocality as an Axiom. Foundations of Physics, 24, 379-385. https://doi.org/10.1007/BF02058098

[3] Clauser, J.F., Horne, M.A., Shimony, A. and Holt, R.A. (1969) Proposed Experiment to Test Local Hidden-Variable Theories. Physical Review Letters, 23, 880-884. https://doi.org/10.1103/PhysRevLett.23.880

[4] Bell, J.S. (1987) Speakable and Unspeakable in Quantum Mechanics. Cambridge University Press, Cambridge.

[5] Cirel'son, B.S. (1980) Quantum Generalizations of Bell's Inequality. Letters in Mathematical Physics, 4, 93-100. https://doi.org/10.1007/BF00417500 
[6] van Dam, W. (2005) Implausible Consequences of Superstrong Nonlocality. arXiv: quant-ph/0501159.

[7] Bub, J. (2012) Why the Tsirelson Bound? In: Ben-Menahem, Y. and Hemmo, M., Eds., Probability in Physics, The Frontiers Collection, Springer, Berlin, Heidelberg, 167-185. https://doi.org/10.1007/978-3-642-21329-8_11

[8] Einstein, A. (1948) Quanten-Mechanik und Wirklichkeit. Dialectica, 2, 320-324. (Translation Howard, 1985)

[9] Pawlowski, M.P., Paterek, T., Kaszlikowski, D., Scarani, V., Winter, A. and Zukowski, M. (2010) Information Causality as a Physical Principle. Nature, 461, 1101-1104.

[10] Masanes, L. (2003) Necessary and Sufficient Conditions for Quantum-Generated Correlations. arXiv:0309137v1.

[11] Navascués, M., Pironio, S. and Acin, A. (2007) Bounding the Set of Quantum Correlations. Physical Review Letters, 98, 010401. https://doi.org/10.1103/PhysRevLett.98.010401

[12] Ishizaka, S. (2017) Cryptographic Quantum Bound on Nonlocality. Physical Review $A$, 95, 022108. https://doi.org/10.1103/PhysRevA.95.022108

[13] Allcock, J., Brunner, N., Pawlowski, M. and Scarani, V. (2009) Recovering Part of the Quantum Boundary from Information Causality. arXiv:0906.3464v3. 